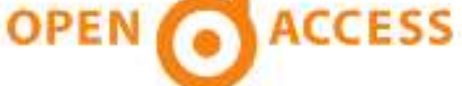

\section{International Journal of Applied Sciences and Biotechnology}

\section{A Rapid Publishing Journal}

ISSN 2091-2609

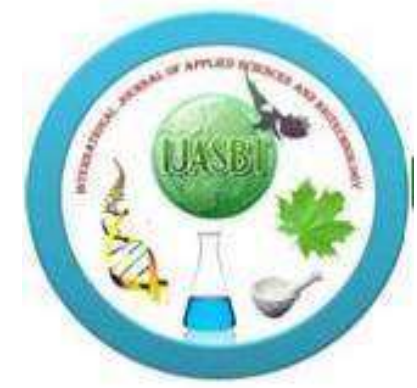

\section{Available online at:}

http://www.ijasbt.org

$\&$

http://www.nepjol.info/index.php/IJASBT/index

\section{Indexing and Abstracting}

CrossRef, Google Scholar, Global Impact Factor, Genamics, Index Copernicus, Directory of Open Access Journals, WorldCat, Electronic Journals Library (EZB), Universitätsbibliothek Leipzig, Hamburg University, UTS (University of Technology, Sydney): Library, International Society of Universal Research in Sciences (EyeSource), Journal Seeker, WZB, Socolar, BioRes, Indian Science, Jadoun Science, Jour-Informatics, Journal Directory, JournalTOCs, Academic Journals Database, Journal Quality Evaluation Report, PDOAJ, Science Central, Journal Impact Factor, NewJour, Open Science Directory, Directory of Research Journals Indexing, Open Access Library, International Impact Factor Services, SciSeek, Cabell's Directories, Scientific Indexing Services, CiteFactor, UniSA Library, InfoBase Index, Infomine, Getinfo, Open Academic Journals Index, HINARI, etc.

\section{CODEN (Chemical Abstract Services, USA): IJASKD}

Vol-2(4) December, 2014
Impact factor*: $\mathbf{1 . 4 2 2}$

Scientific Journal Impact factor\#: $\mathbf{3 . 4 1 9}$

Index Copernicus Value: $\mathbf{6 . 0 2}$

\section{SEM-Biotech}

Publishing

*Impact factor is issued by Universal Impact Factor. Kindly note that this is not the IF of Journal Citation Report (JCR). 


\title{
Research Article \\ BIOINFORMATICS STUDY ON ZAIRE EBOLAVIRUS (EBOV) PROTEIN FOR BETTER UNDERSTANDING THE VACCINE DEVELOPMENT
}

\author{
Mundaganur D.S ${ }^{1}$, Mundaganur D.Y ${ }^{2}$ and Ashokan Kannarath ${ }^{3}$ \\ ${ }^{1}$ Department of zoology, Willingdon College, Sangli, India \\ ${ }^{2}$ Department of Zoology, Miraj Mahavidyalaya, Miraj, India \\ ${ }^{3}$ Department of Zoology, P.V.P.College, K.Mahankal, Sangli, Maharashtra, India-416405 \\ Corresponding author's email: akvsangli@gmail.com
}

\begin{abstract}
Nine, Ebola viruse EBOV (Zaire ebolavirus), proteins are extracted from the NCBI repository and their study was carried out. The physicchemical properties and evolutionary link with other such viruses by homology modeling were carried out. All the proteins show rich in leucine domain an ideal requirement for fast attachment of the virus to the receptor molecule on the host cell surface. The prediction of trans-membrane sequence for the entire glycoprotein component reveals the ability of the virus to enter the host with ease. The lack of adequate homology model for the viral proteins indicates its novel origin and lack of well traceable evolutionary link. We studied the homology based model by using various available tools and find similar approach in all, hence finally concentrated only on one method. The model predicted shows well acceptable region on Ramchandran plot. This discrepancy is only due to the fact that we validate the model to Ramchandran plot and the model predicted were not under the well acceptable 'e' value range i.e. $>1$. Therefore we suggests that vaccine production against this deadly virus should be concentrated on the structure and functions of glycoprotein like low quality secreted glycoprotein (NP_066248), low quality spike glycoprotein(NP_066246)0, small secreted glycoprotein (NP_066247) and RNA dependent RNA polymerase (NP_066251).
\end{abstract}

Key words: Ebola virus (EBOV); Zaire ebolavirus; Protein; Amino acid composition; Homology Model; Ramchandran plot; vaccine.

\section{Introduction}

Ebola virus is a single stranded RNA virus having filamentous structure. Ebola first appeared in Nazara, Sudan and Yambuka, Democratic republic of Congo simultaneously in 1976. The Yambuka is a village near the Ebola river hence the name ebola. The genus ebola consists of five species- Bundibugyo ebolavirus (BDBV), Zaire ebolavirus (EBOV), Reston ebolavirus (RESTV), Sudan ebolavirus (SUDV) and Tai forest ebolavirus (TAFV). The EBOV an SUV is mainly associated with ebola outbreaks in Africa, while RSTV and TAFV is not associated with any ebola outbreak (WHO, 2014). The viral replication needs RNA dependent RNA polymerase and is performed by viral RNA dependent RNA polymerase only (Volchov et al, 1999). The ZEBOV consists of linearly arranged seven genes. These genes are arranged as 3'-NP-VP35-VP40-GPVP30-VP24-L. Order (Volchov et al, 1999). The start and stop codon for transcription is 3'-UAAUU (Volchov et al, 1999). Between the NP and VP35 genes have the sequence (3'-GAU) and (3'-AGO) VP40 and GP genes and 142 bases separate the VP30 and VP24 genes (Volchkov et al, 1999). The 5 end of the leading strand shows stem and loop structure. Structural proteins of Ebolavirus and Marburgvirus contain large regions of homology (Volchkov et $a l, 1999)$. The fatality rate of EBOV is great, about $90 \%$ as compared to other species and death occurs within one day of the infection (Lung et al, 2010). It is observed that a cofactor protein called VP35 is needed for the EBOV RNA polymerase and viral replication (Lung et al, 2010). Virulence s determined by the attachment of the VP35 to the dsRNA.The end cap for VP35 is a hydrophobic molecule. Without the end cap the VP35 cannot attach to the dsRNA (Lung et al, 2010). This end cap helps in the expression of RIG-I-like receptor (RLR). This receptor is the ideal candidate for successful in control in the host immune system. Hence the VP345 protein is the main target for the EBOV treatment. The attack of the EBOV causes to secrete large quantity of chemokines, cytokines, and growth factors and also lymphocyte apoptosis (Warren et al, 2010). Current research also supports the lymphocyte apoptosis theory of disease progress (Waquier et al., 2010). In this paper we attempted to characterize the seven protein including two low quality proteins by bioinformatics tools, servers and software.

\section{Materials and Methods}

In the present study all the proteins available in the NCBI for the search "ebola proteins" was retrieved. The search listed nine proteins as-nucleoprotein (NP_066243), RNA 
dependent-RNA polymerase(NP_066251), matrix protein (NP_066245), low quality secreted glycoprotein (NP_066248), low quality spike glycoprotein(NP_066246)0, small secreted glycoprotein (NP_066247), membrane associated protein(NP_066250), minor nucleoprotein (NP_066249), polymerase glycoprotein (NP_066244). The FASTA sequence was retrieved and used for the further bioinformatics analysis.

\section{Protein statistics}

Protein statistics was studied by using CLC sequence viewer version 6.8.1. The parameter included is sequence information like sequence type, length, weight, isoelctric point, aliphatic index, instability index, GRAVY, half-life, EC; annotations like CDS, protein and regions; amino acid percentages (Ashokan and Pillai, 2008 ).

\section{Homology study}

Homology study was performed by using NCBI protein blast package contain blast-p, psi-blast, phi-blast, delta-blast algorithms, BLOSUM 62 matrix, Existence 11 Extensions1 , with non-redundant protein sequence (nr).

\section{Trans-membrane sequence prediction}

For the prediction of membrane protein SOSUI tools from the repository of EXPASY is used. It was developed for the discrimination of membrane proteins and soluble ones together with the prediction of trans-membrane helices, the accuracy of the classification of proteins was $99 \%$ and the corresponding value for the trans-membrane helix prediction was $97 \%$. The system SOSUI is available through internet access: http://www.tuat.ac.jp/mitaku/sosui/ (Mitaku et al, 2002, Hirokawa et al, 1998). The SOSUI system is a web based tool and users can input their query sequence on the submission section. Results are typically returned in $1 \mathrm{~min}$. Two predictions and two graphs are presented in the output page: (i) the type of protein; (ii) the region of transmembrane helices when the protein is a membrane type; (iii) a graph of the hydropathy plot; (iv) helical wheel diagrams of all trans-membrane helices. Input sequence length is limited to the range of 200-5000 amino acids. Diagrams are displayed by a Java Applet pro- gram (Hirokawa et al, 1998).

\section{D Model predictions}

Protein structure homology modeling has become a routine technique to generate 3D models for proteins when experimental structures are not available (Biasini et al, 2014). SWISS-MODEL is fully automated; with userfriendly web interfaces generate reliable models without the need for complex software packages or downloading large databases. The SWISS-MODEL template library provides annotation of quaternary structure and essential ligands and co-factors to allow for building of complete structural models, including their oligomeric structure (Arnold et al, 2006). The improved SWISS-MODEL pipeline makes extensive use of model quality estimation for selection of the most suitable templates and provides estimates of the expected accuracy of the resulting models. The accuracy of the models generated by SWISS-MODEL is continuously evaluated by the CAMEO system. The new web site allows users to interactively search for templates, cluster them by sequence similarity, structurally compare alternative templates and select the ones to be used for model building. In cases where multiple alternative template structures are available for a protein of interest, a user-guided template selection step allows building models in different functional states. SWISS-MODEL is available at http://swissmodel.expasy.org (Bordoli et al, 2009).

\section{$3 D$ model validation}

For the validation of the generate $\mathrm{d} 3 \mathrm{D}$ model we used VADAR. WASAR (Volume, Area, Dihedral Angle Reporter) is a compilation of more than 15 different algorithms and programs for analyzing and assessing peptide and protein structures from their PDB coordinate data. The results have been validated through extensive comparison to published data and careful visual inspection. The VADAR web server supports the submission of either PDB formatted files or PDB accession numbers. VADAR produces extensive tables and high quality graphs for quantitatively and qualitatively assessing protein structures determined by X-ray crystallography, NMR spectroscopy, 3D-threading or homology modeling (Willard, et al, 2003).

\section{$3 D$ model viewer}

We used Raswin for viewing and changing the pattern of $3 \mathrm{D}$ structure. This software has been created from several sources (Max, 1983, Arthur 1991). Much of the code is from RasMol 2.6, as created by Roger Sayle. The torsion angle code, new POVRAY3 code and other features are derived from the RasMol2.6x1 revisions by Arne Mueller. The Ramchandran printer plot code was derived from fisipl created by Frances C. Bernstein. (Kabsch and Sander, 1983)

\section{Results}

\section{Physical and chemical parameters of the proteins}

The physical and chemical parameters of the proteins were studied by using protparam tool form Expassy (Table1 and 2). All the protein extracted- nucleoprotein (NP_066243), RNA Dependent-RNA polymerase (NP_066251), matrix protein (NP_066245), low quality secreted glycoprotein (NP_066248), low quality spike glycoprotein (NP_066246)0, small secreted glycoprotein (NP_066247), membrane associated protein (NP_066250), minor nucleoprotein (NP_066249), polymerase glycoprotein (NP_066244)- shows invariably 140 amino acids, molecular weight about 16,000, iso-electric point ranges form 5.6-9.4, negatively charged residues range from 10-19, positively charged residues range from 11-25, EC ranges from 11.5 thousands to 30 thousand, half life is invariably 30 hours, instability index ranges from 13.9-39.6, aliphatic index ranges from $70.4-101$ and GRAVY ranges from $0.01-0.4$ 
Table1: Various physical parameters of the protein extracted from EBOV

\begin{tabular}{|c|c|c|c|c|c|c|c|c|c|c|c|c|c|}
\hline $\begin{array}{l}\stackrel{0}{Z} \\
\dot{B}\end{array}$ & 20 & $\begin{array}{l}\frac{\pi}{4} \\
\dot{0} \\
\dot{z}\end{array}$ & $\frac{\overrightarrow{3}}{\dot{0}}$ & $\bar{z}$ & 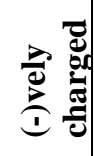 & 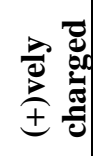 & 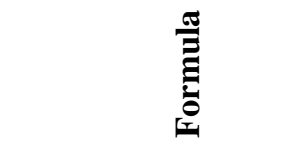 & 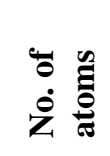 & 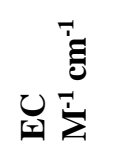 & $\underline{\Xi}$ & 曰 & $z$ & $\frac{1}{3}$ \\
\hline 1 & Nucleoprotein & 140 & 15915 & 5.8 & 19 & 16 & $\mathrm{C}_{712} \mathrm{H}_{1127} \mathrm{~N}_{191} \mathrm{O}_{208} \mathrm{~S}_{7}$ & 2245 & 13075 & 30 & 55.2 & 94.71 & -0.201 \\
\hline 2 & RNA dependent-RNA polymerase & 139 & 15850 & 6.26 & 15 & 14 & $\mathrm{C}_{716} \mathrm{H}_{1124} \mathrm{~N}_{182} \mathrm{O}_{207} \mathrm{~S}_{8}$ & 2237 & 12295 & 30 & 33.8 & 98.13 & -0.068 \\
\hline 3 & Matrix protein & 140 & 15820 & 8.9 & 11 & 13 & $\mathrm{C}_{718} \mathrm{H}_{1126} \mathrm{~N}_{194} \mathrm{O}_{199} \mathrm{~S}_{5}$ & 2242 & 30480 & 30 & 32.72 & 101 & -0.046 \\
\hline 4 & Low quality secreted glycoprotein & 140 & 15952 & 9.4 & 19 & 25 & $\mathrm{C}_{682} \mathrm{H}_{1102} \mathrm{~N}_{220} \mathrm{O}_{213} \mathrm{~S}_{5}$ & 2222 & 4720 & 30 & 57 & 60.6 & -01.022 \\
\hline 5 & Low quality spike glycoprotein & 139 & 15536 & 9.23 & 13 & 18 & $\mathrm{C}_{696} \mathrm{H}_{1097} \mathrm{~N}_{197} \mathrm{O}_{197} \mathrm{~S}_{5}$ & 2192 & 21220 & 30 & 38.10 & 87.6 & -0.202 \\
\hline 6 & Small secreted glycoprotein & 140 & 15665 & 9.38 & 13 & 19 & $\mathrm{C}_{702} \mathrm{H}_{1109} \mathrm{~N}_{199} \mathrm{O}_{198} \mathrm{~S}_{5}$ & 2213 & 21220 & 30 & 39.6 & 86.93 & -.0229 \\
\hline 7 & Membrane associated protein & 140 & 15665 & 9.4 & 13 & 19 & $\mathrm{C}_{702} \mathrm{H}_{1109} \mathrm{~N}_{199} \mathrm{O}_{198} \mathrm{~S}_{5}$ & 2213 & 21220 & 30 & 39.6 & 86.9 & -0.229 \\
\hline 8 & Minor nucleoprotein & 139 & 14938 & 8.1 & 10 & 11 & $\mathrm{C}_{664} \mathrm{H}_{1059} \mathrm{~N}_{181} \mathrm{O}_{200} \mathrm{~S}_{5}$ & 2109 & 11460 & 30 & 13.9 & 88.4 & -0.035 \\
\hline
\end{tabular}


Table 2: Amino acid composition of various protein extracted from EBOV

\begin{tabular}{|c|c|c|c|c|c|c|c|c|c|c|c|c|c|c|c|c|c|c|c|c|c|c|}
\hline \multicolumn{23}{|c|}{ Amino aids } \\
\hline & $\stackrel{g}{z}$ & $\frac{80}{4}$ & 胥 & 安 & s. & క & $\underset{\mathrm{J}}{\mathrm{Z}}$ & $\vec{b}$ & 亲 & $\cong$ & ב્ّ & 3 & $\stackrel{\vec{E}}{\Sigma}$ & $\frac{\mathscr{2}}{2}$ & 2 & $\stackrel{b}{\ddot{~}}$ & है & E & 5 & $\vec{\pi}$ & 7 & 迆 \\
\hline 1 & $=$ & $\underset{\text { in }}{9}$ & $\overrightarrow{\mathrm{i}}$ & 帛 & $\underset{\sim}{\stackrel{+}{*}}$ & $\stackrel{\vec{\varphi}}{\ddot{b}}$ & $\underset{\infty}{\infty}$ & च̈ & $\hat{\mathrm{i}}$ & $\ddot{m}$ & $\stackrel{\text { g }}{=}$ & $\vec{\varphi}$ & $\stackrel{\varphi}{\dot{m}}$ & $\stackrel{m}{q}$ & ç & प्ँ & $\overrightarrow{\mathrm{i}}$ & "뭉 & ले & $=$ & $\Xi$ & $\Xi$ \\
\hline 2 & $\begin{array}{l}9 \\
\dot{n}\end{array}$ & $\stackrel{\leftrightarrow}{\circ}$ & $\stackrel{\text { ì }}{ }$ & $\stackrel{9}{2}$ & $\stackrel{g}{\dot{g}}$ & $\stackrel{9}{6}$ & $\underset{\text { m. }}{9}$ & 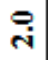 & $\underset{\sim}{\stackrel{i}{i}}$ & $\begin{array}{l}\stackrel{9}{\circ} \\
\dot{n}\end{array}$ & 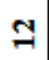 & $\stackrel{9}{\varphi}$ & $\stackrel{\text { تִ }}{ }$ & $\stackrel{g}{\dot{q}}$ & $\stackrel{9}{6}$ & $\stackrel{9}{6}$ & $\stackrel{9}{\circ}$ & $\Xi$ & $\stackrel{9}{9}$ & $\stackrel{9}{\circ}$ & $\Xi$ & $\S$ \\
\hline 3 & $\stackrel{9}{\circ}$ & $\stackrel{g}{g}$ & $\stackrel{\circ}{\circ}$ & $\stackrel{9}{\dot{n}}$ & $\stackrel{9}{6}$ & $\begin{array}{l}\mathscr{S} \\
\dot{n}\end{array}$ & $\stackrel{\overbrace{}}{\mathrm{i}}$ & $\stackrel{9}{\dot{n}}$ & $\stackrel{i}{i}$ & $\stackrel{\theta}{\theta}$ & $\vec{J}$ & $\begin{array}{l}9 \\
\dot{n}\end{array}$ & $\stackrel{\leftrightarrow}{\mathrm{i}}$ & $\stackrel{\leftrightarrow}{\dot{q}}$ & $\underset{\dot{q}}{\stackrel{9}{*}}$ & $\begin{array}{l}9 \\
\dot{n}\end{array}$ & $\stackrel{8}{\circ}$ & 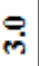 & $\stackrel{\theta}{\rightarrow}$ & $\underset{\dot{q}}{\stackrel{9}{*}}$ & $\Xi$ & $\Xi$ \\
\hline 4 & $\stackrel{9}{5}$ & $\exists$ & 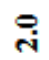 & $\stackrel{\ominus}{\emptyset}$ & $\stackrel{\text { i }}{\mathrm{i}}$ & $\stackrel{9}{\circ}$ & $\stackrel{9}{\circ}$ & $\stackrel{\ominus}{\dot{q}}$ & $\stackrel{\ominus}{\dot{q}}$ & $\stackrel{\vec{i}}{\mathrm{i}}$ & $\stackrel{9}{\circ}$ & $\stackrel{9}{\varphi}$ & 5 & ते & $=$ & $\stackrel{m}{\alpha}$ & هُ & $\Xi$ & $\overrightarrow{\mathrm{i}}$ & : & $\Xi$ & $\Xi$ \\
\hline 5 & $\stackrel{m}{q}$ & $\stackrel{9}{2}$ & $\stackrel{9}{\dot{m}}$ & $\ddot{n}$ & $\hat{\text { ते }}$ & $\hat{i}$ & $\stackrel{m}{q}$ & $\stackrel{\varphi}{\infty}$ & $\stackrel{+}{-}$ & $\begin{array}{l}\ddot{n} \\
\dot{n}\end{array}$ & $\stackrel{m}{\alpha}$ & $\ddot{n}$ & r & $\begin{array}{l}\ddot{n} \\
\dot{n}\end{array}$ & पैं & $=$ & $\stackrel{9}{\circ}$ & $\vec{i}$ & $\vec{i}$ & $\stackrel{m}{\Omega}$ & $\Xi$ & $\Xi$ \\
\hline 6 & $\stackrel{m}{q}$ & 2 & 象 & 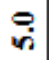 & $\hat{\mathrm{i}}$ & ते & $\stackrel{m}{q}$ & 吕 & ت. & $\stackrel{9}{\text { m. }}$ & $\stackrel{m}{a}$ & $\stackrel{r}{m}$ & $\stackrel{9}{\circ}$ & $\stackrel{9}{n}$ & ¿ैं & $=$ & m & $\vec{i}$ & $\vec{i}$ & $\stackrel{m}{a}$ & $\Xi$ & $\S$ \\
\hline 7 & 車 & : & مُ & $\stackrel{m}{q}$ & $\Xi$ & تُ. & $\hat{\mathrm{i}}$ & ?̧ & กิ & 2 & $\stackrel{Y}{2}$ & $\hat{\imath}$ & ले & $\hat{~}$ & ğ & 苛 & ğ & $\stackrel{5}{g}$ & $\hat{\mathrm{i}}$ & $\stackrel{1}{*}$ & $\Xi$ & g \\
\hline 8 & $\ddot{\varphi}$ & : & $\stackrel{r}{n}$ & ம் & $\hat{\mathrm{N}}$ & هo & $\vec{v}$ & $\stackrel{9}{\vec{n}}$ & $\stackrel{+}{-}$ & $\ddot{n}$ & घे & $\begin{array}{l}\varphi \\
\text { ले }\end{array}$ & $\stackrel{m}{q}$ & $\stackrel{\square}{-}$ & $\dot{m}$ & $\underset{\infty}{\infty}$ & $\dot{7}$ & 5 & $\underset{-}{+}$ & $\ddot{n}$ & $\Xi$ & $\Xi$ \\
\hline 9 & $\stackrel{\ominus}{\emptyset}$ & $\stackrel{9}{\sin }$ & ஸุ & r & लें & $\stackrel{\infty}{\infty}$ & भु & $\stackrel{9}{\dot{m}}$ & $\stackrel{m}{n}$ & $\begin{array}{l}\varphi \\
\dot{n}\end{array}$ & $\vec{\varphi}$ & भy & $\underset{d}{g}$ & $\stackrel{\text { İ }}{-}$ & $\begin{array}{l}\varphi \\
\dot{n}\end{array}$ & $\stackrel{m}{\infty}$ & $\exists$ & $\stackrel{5}{\circ}$ & $\stackrel{m}{n}$ & में & $\Xi$ & § \\
\hline
\end{tabular}

Table 3: Homology parameters for the proteins studies

\begin{tabular}{|c|c|c|c|c|}
\hline Protein & $\begin{array}{c}\text { Accession } \\
\text { number }\end{array}$ & Homology & $\%$ & E-value \\
\hline \multirow{3}{*}{ Nucleoprotein } & \multirow{3}{*}{ (NP_066243) } & Marburg marburgirus & 33 & $7 e-07$ \\
\hline & & $\begin{array}{l}\text { Raven virus - Raven, } \\
\text { Kenya, } 1987\end{array}$ & 33 & $7 e-07$ \\
\hline & & $\begin{array}{l}\text { Lake Victoria } \\
\text { marburgvirus - DRC1999 }\end{array}$ & 33 & $7 \mathrm{e}-07$ \\
\hline \multirow{2}{*}{$\begin{array}{l}\text { RNA } \\
\text { dependent-RNA } \\
\text { polymerase }\end{array}$} & \multirow{2}{*}{ (NP_066251) } & Bundibugyo ebolavirus & 75 & $7 e-67$ \\
\hline & & Marburg marburgirus & 45 & $7 e-24$ \\
\hline \multirow{3}{*}{ Matrix protein } & \multirow{3}{*}{ (NP_066245) } & Bundibugyo ebolavirus & 87 & $7 e-86$ \\
\hline & & Marburg marburgirus & 41 & $7 e-24$ \\
\hline & & $\begin{array}{l}\text { Lake Victoria } \\
\text { marburgvirus - Angola2005 }\end{array}$ & 41 & $8 \mathrm{e}-24$ \\
\hline \multirow{2}{*}{$\begin{array}{l}\text { Low quality } \\
\text { secreted } \\
\text { glycoprotein }\end{array}$} & \multirow{2}{*}{ (NP_066248) } & $\begin{array}{l}\text { Lake Victoria } \\
\text { marburgvirus - Leiden }\end{array}$ & 41 & 8e-09 \\
\hline & & Marburg marburgirus & 56 & $7 e-07$ \\
\hline $\begin{array}{l}\text { Low quality } \\
\text { spike } \\
\text { glycoprotein }\end{array}$ & (NP_066246) & Lloviu cuevavirus & 58 & $7 e-39$ \\
\hline $\begin{array}{l}\text { Small secreted } \\
\text { glycoprotein }\end{array}$ & (NP_066247) & Lloviu cuevavirus & 58 & $7 e-39$ \\
\hline $\begin{array}{l}\text { Membrane } \\
\text { associated } \\
\text { protein } \\
\end{array}$ & (NP_066250) & Lloviu cuevavirus & 58 & $7 e-39$ \\
\hline $\begin{array}{l}\text { Minor } \\
\text { nucleoprotein }\end{array}$ & (NP_066249) & Marburg marburgirus & 41 & $9 e-10$ \\
\hline $\begin{array}{l}\text { Polymerase } \\
\text { glycoprotein }\end{array}$ & (NP_066244) & Reston ebolavirus - Reston & 48 & $8 e-24$ \\
\hline
\end{tabular}


The chemical parameters (Table 2) shows phenyl alanine and serine is totally absent in all the proteins studied, tryptophan lacks in RNA dependent-RNA polymerase (NP_066251 and cystein lacks in Membrane associated protein. Lucien is the maximum in nucleoprotein in alal the nine proteins studied in EBOV.

The homology model study (Table 3 ) shows nucleoprotein is more related to Marburg marburgvirus, RNA dependentRNA polymerase to Bundibugyo ebolavirus and Marburg marburgvirus, , matrix protein also to Bundibugyo ebolavirus and Marburg marburgvirus, low quality secreted glycoprotein to Marburg marburgvirus, low quality spike glycol-protein to, small secreted glycoprotein and membrane associated protein to Lloviu cuevavirus, minor nucleoprotein to Marburg marburgvirus, and polymerase glycoprotein to Reston ebolavirus - Reston.

Trans-membrane protein analysis shows (Table 4) it is absent in Nucleoprotein, Matrix protein, Low quality secreted glycoprotein, Minor nucleoprotein and Polymerase glycoprotein. All the other four proteins show transmembrane sequence. Out of these four proteins RNA dependent-RNA polymerase shows (LSDVPVATLPIDFIVPVLLKALS) different sequence from Low quality spike glycoprotein, Small secreted glycoprotein and Membrane associated protein (TSFFLWVIILFQRTFSIPLGVIH). But in all the proteins which exhibit trans-membrane shows sequence of length 23 amino-acids starting from $17^{\text {th }} \mathrm{N}$-terminal and end in $39^{\text {th }} \mathrm{C}$ terminal. TM in RNA dependent-RNA polymerase shows begins from $63^{\text {rd }} \mathrm{N}$-terminal and end in $85^{\text {th }} \mathrm{C}$-terminal.

The 3D model (Table 5 and Fig. 1) analysis shows that all the nine protein has no exact and satisfactory model with QMEAN > 1. The protein Low quality secreted glycoprotein has no model not at all available in the repository. But the Validity study of the model shows al the models are well in the acceptable level as in the Ramchandran plot. (Fig.2).

Table 3: Homology parameters for the proteins studies

\begin{tabular}{|c|c|c|c|c|}
\hline Protein & $\begin{array}{c}\text { Accession } \\
\text { number }\end{array}$ & Homology & $\%$ & E-value \\
\hline \multirow{3}{*}{ Nucleoprotein } & \multirow{3}{*}{ (NP_066243) } & Marburg marburgivis & 33 & $7 e-07$ \\
\hline & & $\begin{array}{l}\text { Raven virus - Raven, } \\
\text { Kenya, } 1987\end{array}$ & 33 & $7 e-07$ \\
\hline & & $\begin{array}{l}\text { Lake Victoria } \\
\text { marburgvirus - DRC1999 }\end{array}$ & 33 & $7 e-07$ \\
\hline \multirow{2}{*}{$\begin{array}{l}\text { RNA } \\
\text { dependent-RNA } \\
\text { polymerase }\end{array}$} & \multirow{2}{*}{ (NP_066251) } & Bundibugyo ebolavirus & 75 & $7 e-67$ \\
\hline & & Marburg marburgivirus & 45 & $7 e-24$ \\
\hline \multirow{3}{*}{ Matrix protein } & \multirow{3}{*}{ (NP_066245) } & Bundibugyo ebolavirus & 87 & $7 e-86$ \\
\hline & & Marburg marburgivis & 41 & $7 e-24$ \\
\hline & & $\begin{array}{l}\text { Lake Victoria } \\
\text { marburgvirus - Angola2005 }\end{array}$ & 41 & $8 e-24$ \\
\hline \multirow{2}{*}{$\begin{array}{l}\text { Low quality } \\
\text { secreted } \\
\text { glycoprotein }\end{array}$} & \multirow{2}{*}{ (NP_066248) } & $\begin{array}{l}\text { Lake Victoria } \\
\text { marburgvirus - Leiden }\end{array}$ & 41 & $8 e-09$ \\
\hline & & Marburg marburgvirus & 56 & $7 e-07$ \\
\hline $\begin{array}{l}\text { Low quality } \\
\text { spike } \\
\text { glycoprotein }\end{array}$ & (NP_066246) & Lloviu cuevavirus & 58 & $7 e-39$ \\
\hline $\begin{array}{l}\text { Small secreted } \\
\text { glycoprotein }\end{array}$ & (NP_066247) & Lloviu cuevavirus & 58 & $7 e-39$ \\
\hline $\begin{array}{l}\text { Membrane } \\
\text { associated } \\
\text { protein }\end{array}$ & (NP_066250) & Lloviu cuevavirus & 58 & $7 e-39$ \\
\hline $\begin{array}{l}\text { Minor } \\
\text { nucleoprotein }\end{array}$ & (NP_066249) & Marburg marburgivirus & 41 & $9 e-10$ \\
\hline $\begin{array}{l}\text { Polymerase } \\
\text { glycoprotein }\end{array}$ & (NP_066244) & Reston ebolavirus - Reston & 48 & $8 e-24$ \\
\hline
\end{tabular}


Table 4: Transmembrane sequence of the protein extracted from EBOV

\begin{tabular}{|c|c|c|c|c|c|c|}
\hline Sl.No & Proteins & $\begin{array}{c}\mathrm{N} \\
\text { termina } \\
\mathrm{l}\end{array}$ & transmembrane region & $\begin{array}{c}\mathrm{C} \\
\text { termina } \\
\mathrm{l}\end{array}$ & Type & $\begin{array}{c}\text { leng } \\
\text { th }\end{array}$ \\
\hline 1 & Nucleoprotein & \multicolumn{5}{|c|}{ No Trans membrane protein } \\
\hline 2 & $\begin{array}{l}\text { RNA } \\
\text { dependent-RNA } \\
\text { polymerase }\end{array}$ & 63 & $\begin{array}{c}\text { LSDVPVATLPIDFIVPVL } \\
\text { LKALS }\end{array}$ & 85 & $\begin{array}{c}\text { Primar } \\
\mathrm{y}\end{array}$ & 23 \\
\hline 3 & Matrix protein & \multicolumn{5}{|c|}{ No Trans membrane protein } \\
\hline 4 & $\begin{array}{l}\text { Low quality } \\
\text { secreted } \\
\text { glycoprotein }\end{array}$ & \multicolumn{5}{|c|}{ No Trans membrane protein } \\
\hline 5 & $\begin{array}{l}\text { Low quality } \\
\text { spike } \\
\text { glycoprotein } \\
\end{array}$ & 17 & $\begin{array}{c}\text { TSFFLWVIILFQRTFSIP } \\
\text { LGVIH }\end{array}$ & 39 & $\begin{array}{c}\text { Primar } \\
\mathrm{y}\end{array}$ & 23 \\
\hline 6 & $\begin{array}{l}\text { Small secreted } \\
\text { glycoprotein }\end{array}$ & 17 & $\begin{array}{c}\text { TSFFLWVIILFQRTFSIP } \\
\text { LGVIH }\end{array}$ & 39 & $\begin{array}{c}\text { Primar } \\
y\end{array}$ & 23 \\
\hline 7 & $\begin{array}{l}\text { Membrane } \\
\text { associated } \\
\text { protein }\end{array}$ & 17 & $\begin{array}{c}\text { TSFFLWVIILFQRTFSIP } \\
\text { LGVIH }\end{array}$ & 39 & $\begin{array}{c}\text { Primar } \\
\mathrm{y}\end{array}$ & 23 \\
\hline 8 & $\begin{array}{l}\text { Minor } \\
\text { nucleoprotein }\end{array}$ & \multicolumn{5}{|c|}{ No Trans membrane protein } \\
\hline 9 & $\begin{array}{l}\text { Polymerase } \\
\text { glycoprotein }\end{array}$ & \multicolumn{5}{|c|}{ No Trans membrane protein } \\
\hline
\end{tabular}

Table 5: 3D model Parameters deducted for various proteins assessed for EBOV

\begin{tabular}{|c|c|c|c|c|c|c|c|c|}
\hline No & Protein & Template & $\begin{array}{l}\text { Identity } \\
\text { In \% }\end{array}$ & Description & QMOE & $\begin{array}{l}\text { QMEAN } \\
4\end{array}$ & $\begin{array}{l}\text { Oligo- } \\
\text { state }\end{array}$ & $\begin{array}{l}\text { Ligand } \\
\mathrm{s}\end{array}$ \\
\hline 1 & Nucleoprotein & 4bk9.1.A & 16.13 & $\begin{array}{l}\text { 2-DEHYDRO-3- } \\
\text { DEOXYPHOSPHOGL } \\
\text { UCONATE } \\
\text { ALDOLASE/4- } \\
\text { HYDROXY-2-OXO } \\
\text { GLUTARATE } \\
\text { ALDOLASE }\end{array}$ & 0.10 & -0.70 & $\begin{array}{l}\text { MON } \\
\text { OME } \\
\text { R }\end{array}$ & None \\
\hline 2 & $\begin{array}{l}\text { RNA } \\
\text { dependent- } \\
\text { RNA } \\
\text { polymerase }\end{array}$ & 4dkw.l.A & 25.93 & $\begin{array}{l}\text { Large terminase } \\
\text { protein }\end{array}$ & 0.08 & -0.07 & $\begin{array}{l}\text { MON } \\
\text { OME } \\
\text { R } \\
\end{array}$ & None \\
\hline 3 & $\begin{array}{l}\text { Matrix } \\
\text { protein }\end{array}$ & 4m0q.1.B & 100 & $\begin{array}{l}\text { Membrane-associated } \\
\text { protein VP24 }\end{array}$ & 0.98 & -1.92 & $\begin{array}{l}\text { Homo- } \\
\text { dimer }\end{array}$ & None \\
\hline 4 & $\begin{array}{l}\text { Low quality } \\
\text { secreted } \\
\text { glycoprotein }\end{array}$ & Nil & Nil & sols & Nil & Nil & Nil & Nil \\
\hline 5 & $\begin{array}{l}\text { Low quality } \\
\text { spike } \\
\text { glycoprotein }\end{array}$ & 3ve0.1.A & 81.48 & Envelope glycoprotein & 0.75 & -2.34 & $\begin{array}{l}\text { MON } \\
\text { OME } \\
\text { R } \\
\end{array}$ & Non \\
\hline 6 & $\begin{array}{l}\text { Small } \\
\text { secreted } \\
\text { glycoprotein }\end{array}$ & 3s88.1.B & 81.65 & Envelope glycoprotein & 0.74 & -2.00 & $\begin{array}{l}\text { MON } \\
\text { OME } \\
\text { R }\end{array}$ & Non \\
\hline 7 & $\begin{array}{l}\text { Membrane } \\
\text { associated } \\
\text { protein }\end{array}$ & 3s88.1.B & 81.65 & Envelope glycoprotein & 0.74 & -2.00 & $\begin{array}{l}\text { MON } \\
\text { OME } \\
\text { R } \\
\end{array}$ & Non \\
\hline 8 & $\begin{array}{l}\text { Minor } \\
\text { nucleoprotein }\end{array}$ & 4ld8.1.A & 86.60 & Matrix protein VP40 & 0.72 & -0.88 & $\begin{array}{l}\text { Homo- } \\
\text { dimer }\end{array}$ & None \\
\hline 9 & $\begin{array}{l}\text { Polymerase } \\
\text { glycoprotein }\end{array}$ & 3nmd.1.B & 20.93 & $\begin{array}{l}\text { cGMP Dependent } \\
\text { Protein Kinase }\end{array}$ & 0.15 & -0.96 & $\begin{array}{l}\text { MON } \\
\text { OME } \\
\text { R }\end{array}$ & None \\
\hline
\end{tabular}



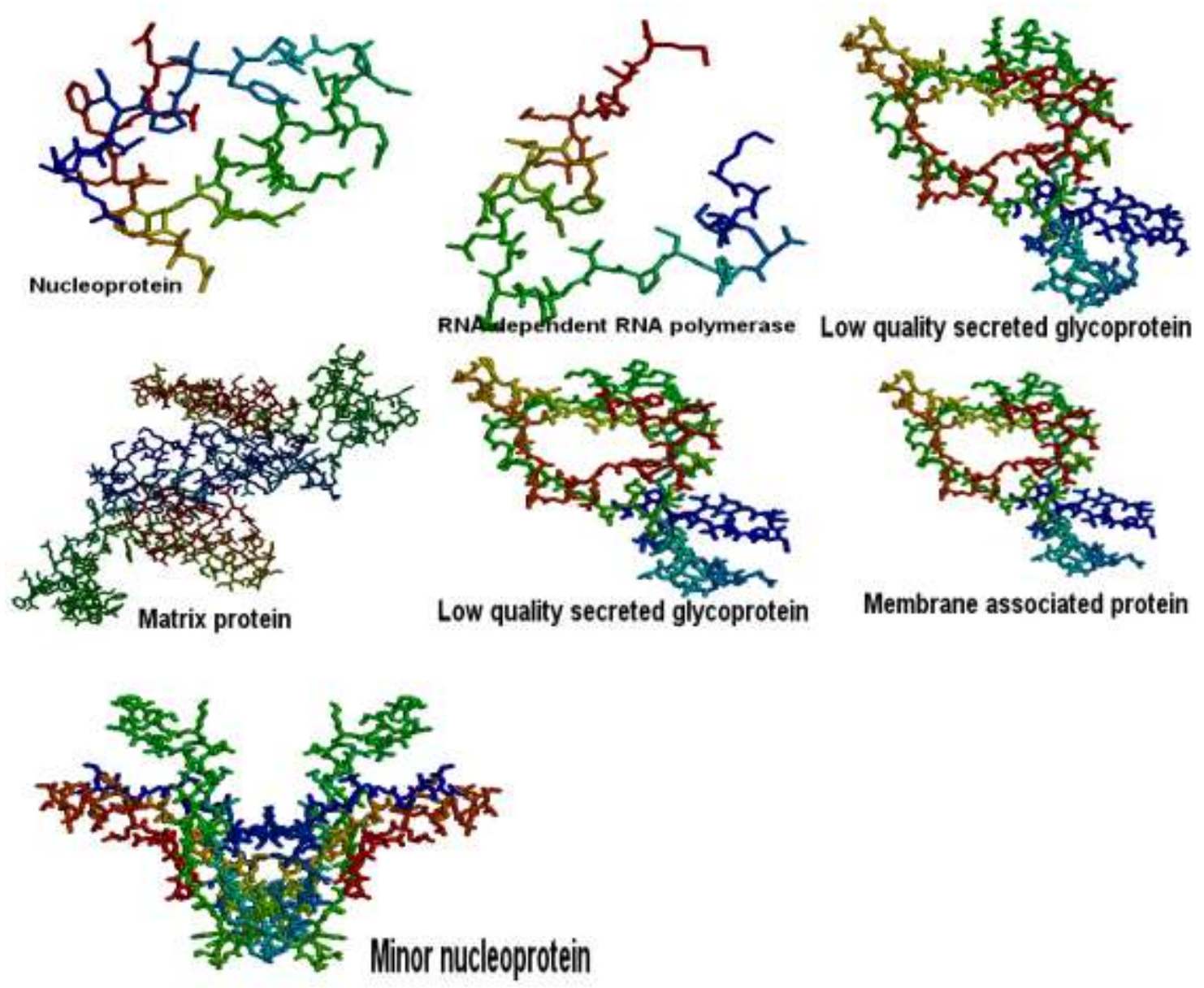

Fig. 1: 3D Model generated for various proteins of EBOV
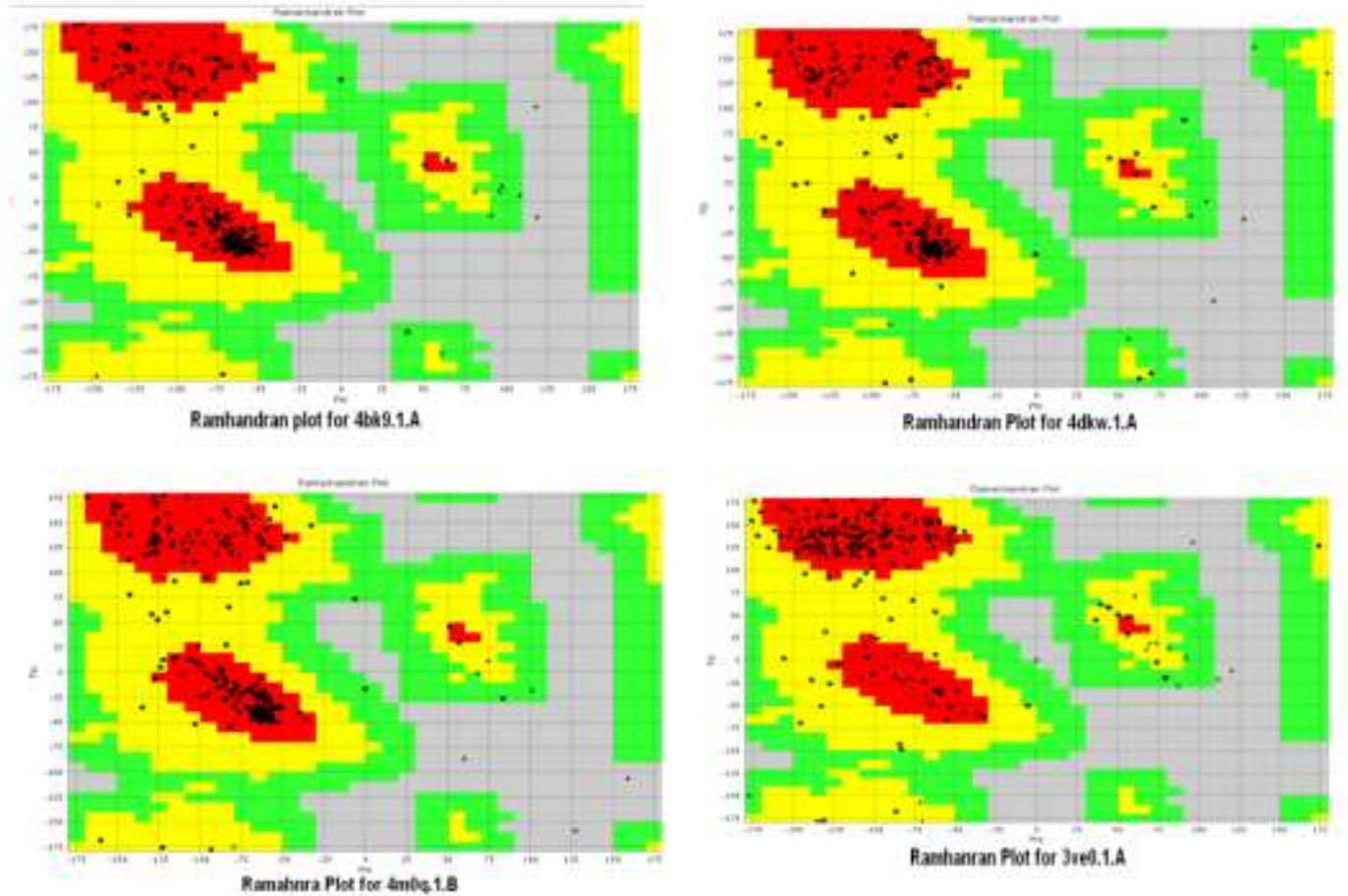

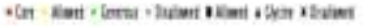

Fig. 2: Ramchandran Plot generated for selected EBOV proteins against the pdb structure 


\section{Discussion}

Ebola hemorrhagic fever is a severe and often deadly illness that can occur in humans and primates. It made worldwide news because of its destructive potential. It is caused by a virus belonging to the family called Filoviridae. Scientists have identified five types of Ebola virus. Four have been reported to cause disease in humans: Ebola-Zaire virus, Ebola-Sudan virus, Ebola-Ivory Coast virus, and EbolaBundibugyo. The human disease has so far been limited to parts of Africa. The Reston type of Ebola virus has recently been found in the Philippines. The disease can be passed to humans from infected animals and animal materials. Ebola can also be spread between humans by close contact with infected body fluids or through infected needles in the hospital. During the incubation period, which can last about 1 week (rarely up to 2 weeks) after infection, symptoms include: Arthritis, Backache (low-back pain) ,Chills, Diarrhea, Fatigue, Fever, Headache, Malaise, Nausea, Sore throat, Vomiting. Late symptoms include:, Bleeding from eyes, ears, and nose, Bleeding from the mouth and rectum (gastrointestinal bleeding), Eye swelling (conjunctivitis), Genital swelling (labia and scrotum), Increased feeling of pain in the skin, Rash over the entire body that often contains blood (hemorrhagic), Roof of mouth looks red (Bausch, 2011, Peters et al, 2009). The present investigation is concentrated on nine available protein sequences in the repository of NCBI. The physical parameter shows that all proteins are small with amino acids about 140 in number having molecular weight around 16,000 . Along with these data and iso-electric point value will enable the researchers to separate the protein with little efforts. All the proteins are rich in leucine domain, it may be related with to increase the affinity to attach strongly to the host cell surface receptor as described by Luo et al, 1999. The homology study shows that EBOV is more related to the Marburg marburgvirus, except the proteins low quality spike glycoprotein (NP_066246)0, small secreted glycoprotein (NP_066247), membrane associated protein (NP_066250) these are more related with Lloviu cuevavirus, thus a bi-focal approach may be needed to treat the virus and to control the infection. All the glycoprotein shows trans-membrane sequences identical, hence a common vaccine or medicine may in the future can help to control the disease in effective manner. The lack of exact matches in the sense of ' $e$ ' value shows that the virus is new and has no evolutionary link with other such virus. This phylogenic relation is to be clarified in a better way. The more acceptable regions in the ramchndarn plot is only due to the fact that we assess the validity of the predicted model with ramchandran plot, hence concerned to the model the plot is valued and not with the structure of the viral protein.

\section{References}

Altschul SF, Gish W, Miller W, Myers EW and Lipman DJ (1990) Basic local alignment search tool. J. Mol. Biol. 215:403410. DOI: $10.1016 / \mathrm{S} 0022-2836(05) 80360-2$

Arnold K, Bordoli L, Kopp J, and Schwede T (2006) The SWISSMODEL Workspace: A web-based environment for protein structure homology modeling. Bioinformatics 22:195-201. DOI: 10.1093/bioinformatics/bti770

Arthur ML (1991) Protein Architecture: A Practical Approach, IRL Press Publishers,.

Ashokan KV and Pilali MM (2008) In silico characterization of silk fibroin protein using computatational tools and servers. Asian Journal of experimental sciences 22(3): 265-274.

Bausch DG (2011) Viral hemorrhagic fevers. In: Goldman L, Ausiello D (eds.) Goldman's Cecil Medicine. 24th ed. Philadelphia, PA: Saunders Elsevier; chap 389.

Biasini M, Bienert S, Waterhouse A, Arnold K, Studer G, Schmidt T, Kiefer F, Gallo T, Bertoni C.M, Bordoli L and Schwede $\mathrm{T}$ (2014). SWISS-MODEL: modeling protein tertiary and quaternary structure using evolutionary information Nucleic Acids Research 42: 252-258. DOI: 10.1093/nar/gku340

Bordoli L, Kiefer F, Arnold K, Benkert P, Battey J and Schwede $T$ (2009) Protein structure homology modeling using SWISS-MODEL Workspace. Nature Protocols 4: 1. DOI: 10.1038/nprot.2008.197

Camacho C, Coulouris G, Avagyan V, Ma N, Papadopoulos J, Bealer K and Madden TL (2008) BLAST+: architecture and applications. BMC Bioinformatics 10:421. DOI: 10.1186/1471-2105-10-421

Hirokawa T, Boon-Chieng S, and Mitaku S (1998) SOSUI: classification and secondary structure prediction system for membrane proteins. Bioinformatics 14: 378-379. DOI: 10.1093/bioinformatics/14.4.378

Kabsch W and Sander C (1983) Dictionary of Protein Secondary Structure: Pattern Recognition of Hydrogen-Bonded and Geometrical Features. Biopolymers 22:2577-2637. DOI: 10.1002/bip.360221211

Leung W, Shaffer CD, Cordonnier T, Wong J, Itano MS and et al. (2010). Evolution of a Distinct Genomic Domain in Drosophila: Comparative Analysis of the Dot Chromosome in Drosophila melanogaster and Drosophila virilis. Genetics 185(4): 1519-- 1534. DOI: 10.1534/genetics.110.116129

Luo Z, Matthews A.M and Weiss S.R (1999). Amino Acid Substitutions within the Leucine Zipper Domain of the Murine Coronavirus Spike Protein Cause Defects in Oligomerization and the Ability To Induce Cell-to-Cell Fusion. J Virol, 73(10): 8152-8159.

Max N (1983) Computer Representation of Molecular Surfaces, IEEE Computer Graphics and Applications, pp. 21-29. DOI: 10.1109/MCG.1983.263183 
Mitaku S, Hirokawa T and Tsuji T (2002) Amphiphilicity index of polar amino acids as an aid in the characterization of amino acid preference at membrane-water interfaces. Bioinformatics 18: 608-616. DOI: 10.1093/bioinformatics/18.4.608

Morgulis A, Coulouris G, Raytselis Y, Madden TL, Agarwala R. and Schäffer AA (2008) Database indexing for production MegaBLAST searches. Bioinformatics 15:1757-1764. DOI: 10.1093/bioinformatics/btn322

Peters CJ (2009) Marburg and Ebola virus hemorrhagic fevers. In: Mandell GL, Bennett JE, Dolin R (eds.) Principles and Practice of Infectious Diseases. 7th ed. Philadelphia, PA: Churchill Livingstone Elsevier; chap 164.

Sanhz JP, Bouillt MN, Colineau E, Blaise A, Amanowics M, Blurlet P, Fournier JM, Charvolin T and Larroque J (1993) Physica B, 186-188: 675-677.

Schaffer AA, Aravind L, Madden TL, Shavirin S, Spouge JL, Wolf YI, Koonin EV, Altschul SF. (2001) Improving the accuracy of PSI-BLAST protein database searches with composition-based statistics and other refinements. Nucleic Acids Res. 29(14):2994-3005. DOI: 10.1093/nar/29.14.2994
Volchkov VE, Vochkova VA, Chepurnov AA, Blinov VM, Dolink O, Netesov SV and Feldmannn H (1999) Characterization of the L-gene and 5 trailer region of Ebola virus. J. Gen. Virol. 80: 355-362.

Waquier N, Becquart P, Padilla C, Baize S and Leroy EM (2010) Human Fatal Zaire Ebola Virus Infection Is Associated with an Aberrant Innate Immunity and with Massive Lymphocyte Apoptosis. PLOS Neglected Tropical disease 4(10):1-10. DOI: $10.1371 \% 2$ Fjournal.pntd.0000837

Warren L, Manos PD, Ahfeldt T, Loh YH, Li H, Lau F, Ebina W, and et al. (2010). Highly efficient reprogramming to pluripotency and directed differentiation of human cells with synthetic modified mRNA. Cell Stem Cell 7: 618630. DOI: $10.1016 /$ j.stem.2010.08.012

WHO: www.who.int/mediacentre/factsheets/fs103/en/

Willard L, Ranjan A, Zhang H, Monzavi H, Robert FB, Brian DS and David SW (2003) VADAR: a web server for quantitative evaluation of protein structure quality. Nucleic Acids Res. 31 (13): 3316-3319. DOI: $10.1093 /$ nar/gkg565 